\title{
Spatio-temporal Directional Filtering for Improved Inversion of MR Elastography Images
}

\author{
Armando Manduca, David S. Lake, and Richard L. Ehman \\ MRI Research Lab, Mayo Clinic and Foundation, Rochester, MN 55905, USA \\ \{manduca.armando, lake.david,ehman.richard\}@mayo.edu
}

\begin{abstract}
MR elastography can visualize and measure propagating shear waves in tissue-like materials subjected to harmonic mechanical excitation. This allows the calculation of local values of material parameters such as shear modulus and attenuation. Various inversion algorithms to perform such calculations have been proposed, but they are sensitive to areas of low displacement amplitude (and hence low SNR) that result from interference patterns due to reflection and refraction. A spatio-temporal directional filter applied as a preprocessing step can separate interfering waves so they can be processed separately. Weighted combinations of inversions from such directionally separated data sets can significantly improve reconstructions of shear modulus and attenuation.
\end{abstract}

\section{Introduction}

Magnetic resonance elastography (MRE, [1]) uses harmonic mechanical displacements as a probe of the material properties of soft tissues, spatially mapping and measuring motions with amplitudes of $1 \mathrm{~m}$ or less. The resulting "wave images" reflect the displacement of spins due to acoustic strain wave propagation. These data allow the calculation of local values of material parameters such as shear modulus and attenuation, with possible uses ranging from tissue characterization to tumor detection and diagnosis.

Wave images can be obtained at various phase offsets regularly spaced around a motion cycle. This allows extraction of the harmonic component of motion at the frequency of interest, giving the amplitude and the phase (relative to an arbitrary zero point) of the displacement at each point in space [2]. This complex displacement field is the input to the processing techniques described below. A single MRE acquisition captures only a single component of motion. However, the experiment can be repeated with three orthogonal senstitization directions. Thus, the data set acquired by MRE can be very rich: full 3D complex harmonic displacement information can be acquired at MR pixel resolution throughout a 3D volume.

A variety of inversion techniques have been proposed for MRE data. Under certain assumptions (linearity, incompressibility, local homogeneity), the equation of harmonic motion simplifies to the Helmholtz equation [2], and shear modulus is 
essentially determined by simple division of displacement values by their spatial Laplacian. Direct inversion (DI) uses filtered estimates of displacement and its Laplacian to perform such a division at each point. Local Frequency Estimation (LFE) adds the assumption of no attenuation and combines local estimates of instantaneous frequency over several scales. A simpler technique, phase gradient (PG), assumes propagation of a single shear wave, and simply calculates the gradient of its phase. It breaks down with any wave superposition (reflection, interference, etc.), and its use has been limited to specialized situations. By contrast, DI and LFE are based more fundamentally on the equation of motion, and depend simply on the presence of sufficient motion within the local region. Complex interference patterns from reflection, refraction, etc. pose difficulties only if they create areas of low displacement amplitude and hence low SNR. However, such conditions are frequently present in actual data, confounding these algorithms. In this paper, we propose the use of spatio-temporal directional pre-processing filters that separate complex wave fields into components, each of which can be analyzed separately.

\section{Methods}

An MRE experiment typically captures a number of phase offsets (usually 4-8) regularly spaced around a harmonic motion cycle. These can be thought of as snapshots of the wave field in time, although this is a simplification of the physics of the acquisition. Spatio-temporal filters can be designed in frequency space to select portions of the wave field propagating in specific directions. Since the filtering is a linear operation, the output of such filters satisfies the same equation of motion (under the assumptions above) as the original data. However, such filtering can yield simplified wave fields with less interference that can be analyzed more easily, as shown below. Fig. 1 illustrates a sample wave image from a phantom object with four stiffer cylindrical inclusions perpendicular to the slice. Acoustic shear waves are introduced from the top of the image and propagate downward, but reflect off the cylinders and the boundaries of the phantom, giving rise to interference patterns. The effect of selecting waves propagating in the top-down and bottom-up directions from the filtering approach described below is shown in the center and right panels. These data can then be processed individually, and areas of interference are reduced.

The filters used are a product of radial and spatially directional components, and are oriented in time as well. The data is first transformed to a 3D frequency space (2 spatial directions, one temporal). A wave with a particular spatial frequency (denoted as $\mathrm{k}_{\mathrm{y}}$ with no loss of generality, to correspond with the top-down wave in Fig. 1) is represented by two peaks in frequency space: one at $+\mathrm{k}_{\mathrm{y}}$ in the positive temporal halfspace, and the other at $-\mathrm{k}_{\mathrm{y}}$ in the negative temporal half-space). The wave traveling in precisely the opposite direction (bottom-up, in this example) is represented by symmetrically opposite peaks $\left(+\mathrm{k}_{\mathrm{y}}\right.$ in the negative temporal half-space, etc.). The two can thus be easily separated.

The radial components are Butterworth bandpass filters designed to cut off very low and very high frequencies (see profile in Fig. 2). These have been used in the 
past as a pre-processing step: the high frequencies contain only noise, while removing very low frequencies can aid in removing longitudinal wave and bulk motion effects. The image in the left of Fig. 1 has undergone this radial bandpass filtering.

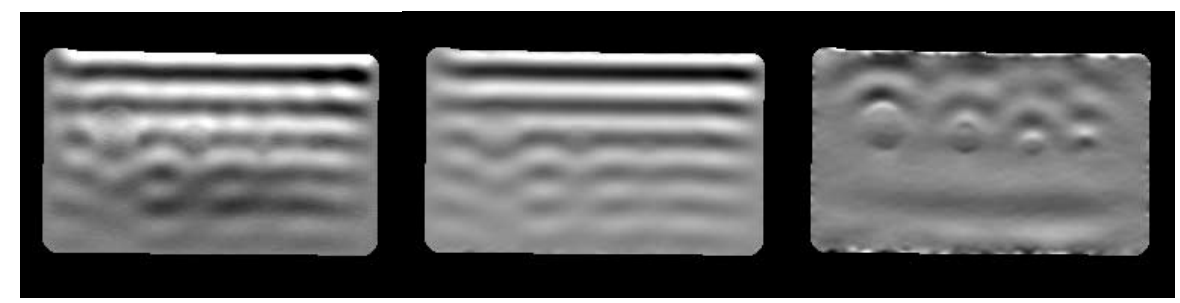

Fig. 1. Left: Original bandpass filtered wave image (for one of eight phase offsets). Center: Data after top-down directional filtering. Right: Data after bottom-up directional filtering

The radial component is then multiplied by a spatial directional component with a $\cos ^{2}$ dependence in the half-space about a selected direction, and zero in the opposite half-space, as illustrated in Fig. 2. This is similar to the orientation dependence of the filters used in the LFE, and has the useful property that, when performed in orthogonal directions, it decomposes the signal into components that can simply be added back together to form the original signal [3]. This filter is applied to the first positive temporal frequency plane $\left(\mathrm{k}_{\mathrm{t}}=+1\right)$. The other temporal frequency planes are discarded: the $\mathrm{k}_{\mathrm{t}}=-1$ plane has conjugate symmetry and thus is not needed; while the other planes contain information at frequencies other than at the mechanical driving frequency that is the frequency of interest. These other harmonics can be filtered and kept if desired, but this is not usually the case.
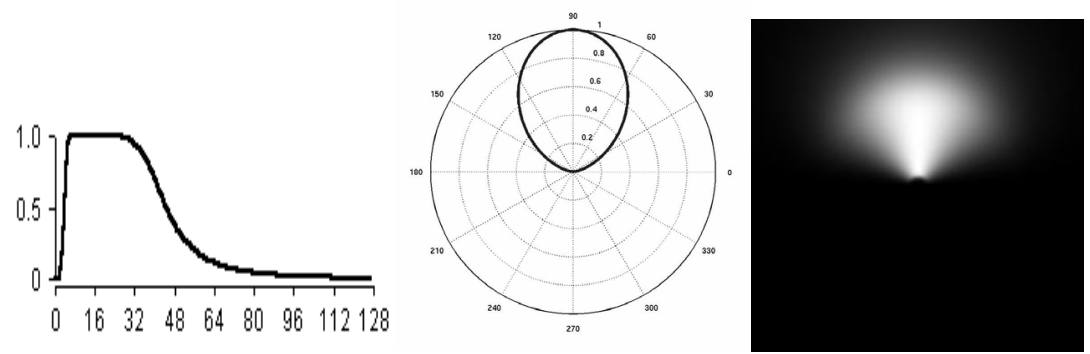

Fig. 2. Left: Profile of the radial component (Butterworth bandpass) component of the filters. Center: Directional component ( $\cos ^{2}$ dependence from the selected direction). The filter is zero in the opposite half-space. Right: The filter in frequency space

An additional example of the decomposition offered by these filters is presented in Fig. 3, for a synthetic data set consisting of waves radiating sinusoidally outward from a central point. Two of the four directional components are shown; all four added together would yield precisely the original data set. 


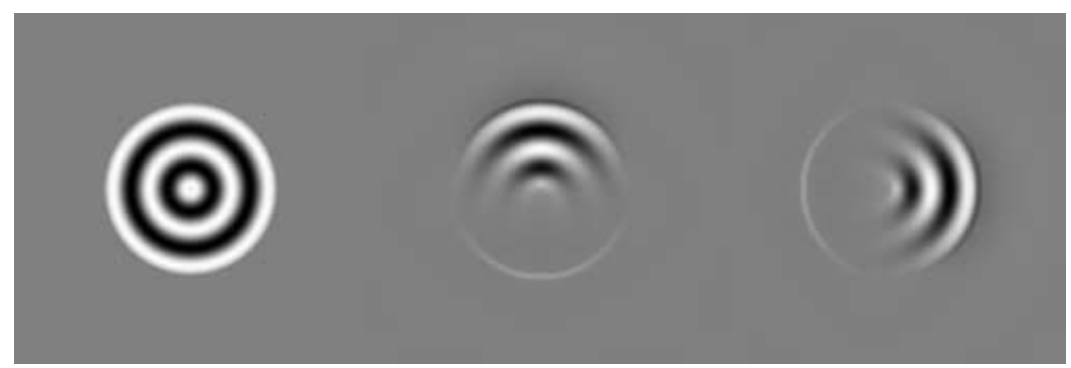

Fig. 3. Left: One sample phase offset of a synthetic data set with waves radiating radially outward. Center: The bottom-to-top directional component as extracted from the filters described. Right: The left-to-right directional component

\section{Results}

Data sets were acquired for a gel phantom with 4 stiff cylindrical inclusions ranging from 5 to $25 \mathrm{~mm}$ in diameter (this is the source of the wave data in Fig. 1). The data were bandpass filtered, and then inversions were performed with all three processing techniques (LFE, DI, PG; Fig. 4, top row). The data were then passed through a topdown directional filter as described above (and illustrated in Fig. 1, center). The inversions were performed again on the directionally filtered data (Fig. 4, bottom).

The effects of directional filtering in Fig. 4 vary strongly with the processing algorithm. The LFE result (left column) shows only minor improvements from the directional filtering; this is to be expected since the filters resemble processing already present in the LFE [3]. The direct inversion (DI) results are significantly improved by directional filtering. The inclusions are sharp and clearly visible, the artifact at region borders are eliminated, and the artifacts in the low amplitude areas with significant interference near the bottom of the image are greatly reduced. Finally, the phase gradient (PG) results are dramatically improved. This technique explicitly assumes a single wave and simply tracks its phase. Since this approach quickly breaks down when this assumption is violated, it has been used only in very restricted situations. The directional filter provides sufficient wave separation that the "single wave" assumption, though still simplistic, is now closer to reality, and the algorithm now yields far more useful results. 


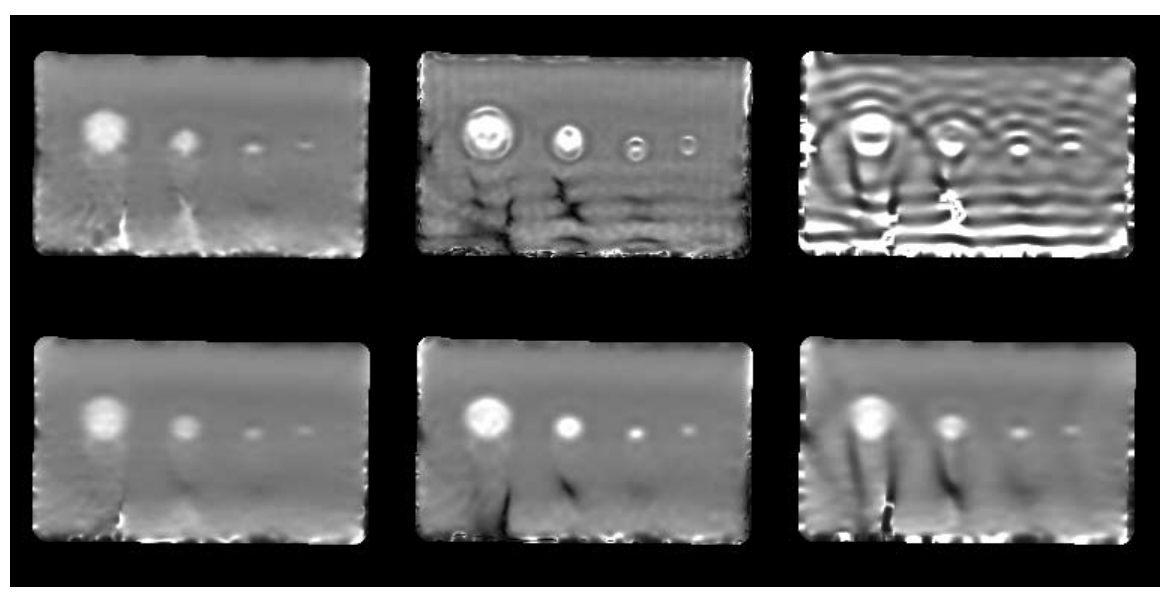

Fig. 4. Top row: The LFE, DI and PG reconstructions of shear modulus from the bandpass filtered data. Bottom row: the LFE, DI and PG reconstructions from the top-down directionally filtered data

The above results use a single top-down directional filter, since that is the main propagation direction in the phantom. In more general applications, especially in vivo, there may be no such preferred direction. In such a case, multiple filters in orthogonal directions may be applied and processed separately, and the results combined, weighted by the relative energy in each filtered data set at each pixel. Fig. 5 confirms that this technique works well on the phantom, even though two of the four directions contain mostly noise. Additionally, one could ask how sensitive the results are to the filter orientations in preferred directions (e.g., the top-down direction in this example). Fig. 5 (right) confirms that the results are essentially isotropic with respect to the filter orientations.

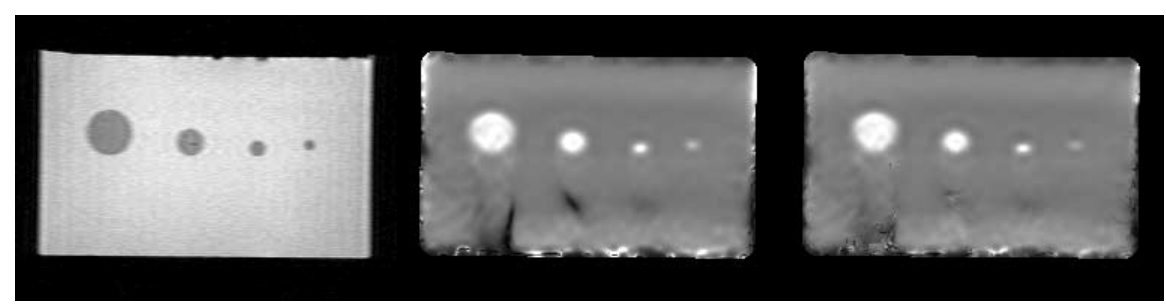

Fig. 5. Left: Magnitude image of phantom. Center: Weighted combination of direct inversion result from four orthogonal directional filters in the horizontal and vertical directions. Right: Combined direct inversion result with four orthogonal filters in the diagonal directions

A more complicated example is shown in Fig. 6. In this case the object is a breast phantom with a stiff inclusion near the center. The driving is upward from the bottom, in a longitudinal (not shear) manner. Mode conversion generates shear waves throughout the object, with significant displacement in all three directions in most parts of the object. The DI reconstruction of shear modulus from data sensitized to $\mathrm{z}$ 
(out of plane) displacement alone is shown in the left panel. The reconstruction is much improved by taking the weighted combination of four reconstructions from four orthogonal directional filters (center panel). At right is the result from additionally combining appropriately weighted reconstructions of directionally filtered data sensitized to displacements in the $\mathrm{x}$ and $\mathrm{y}$ directions (this is now a weighted combination of 12 separate reconstructions). The inclusion is now very well depicted.

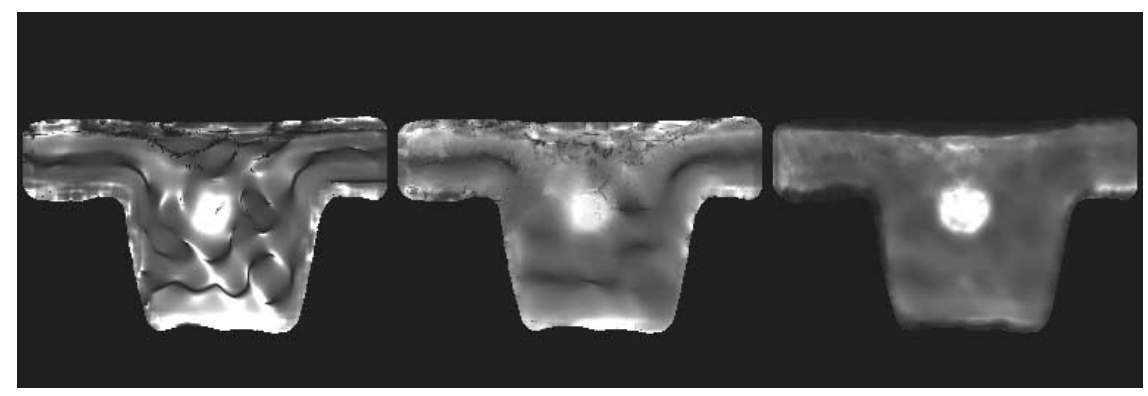

Fig. 6. Left: Direct inversion reconstruction of breast phantom with stiff inclusion near the center with non-directionally filtered displacement data of motion in the $\mathrm{z}$ (out of plane) direction only. Center: Weighted combination of direct inversion result from four orthogonal directional filters in the horizontal and vertical directions. Right: Combined direct inversion result with four orthogonal filters in the diagonal directions

The DI technique also yields attenuation information, but such results typically are noisy, with artificially enhanced boundaries between regions, where the local homogeneity assumption is violated [4]. If directional filtering is applied, such reconstructions in areas away from boundaries are noticeably smoothed. Although artifacts at region boundaries remain, quantitative measurements of attenuation may become possible in sufficiently homogeneous areas (Fig. 7).

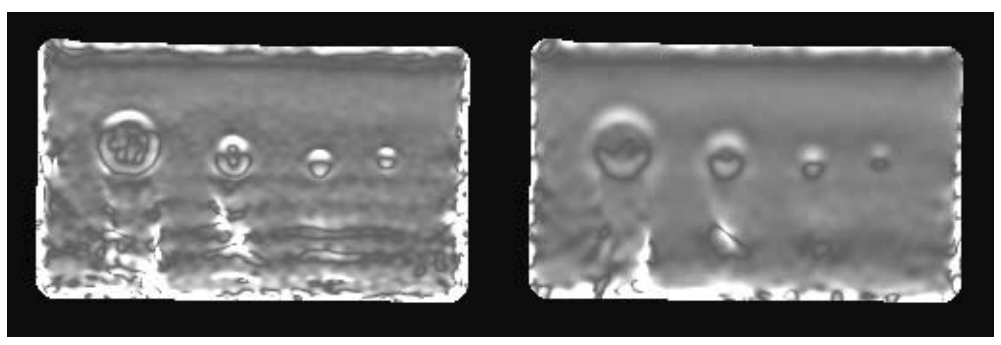

Fig. 7. DI reconstructions of attenuation without (left) and with (right) directional filtering 


\section{Conclusion}

Directional filtering appears to be a very promising pre-processing technique for MRE inversion. By decomposing the wave field into separate components, it reduces the effects of interfering waves, and significantly improves the quality of shear modulus reconstructions. Inversions with the direct inversion and phase gradient algorithms are particularly improved, as are the attenuation maps generated by direct inversion. Orthogonal sets of filters can be combined and appear to handle propagation in arbitrary directions in an isotropic way, obviating any need to specify preferred wave directions. The application of this technique to in vivo data is currently being studied.

\section{References}

1. Muthupillai, R., Lomas, D.J., Rossman, P.J., Greenleaf, J.F., Manduca, A., Ehman, R.L.: Magnetic Resonance Elastography by Direct Visualization of Propagating Acoustic Strain Waves. Science 269 (1995) 1854-1857

2. Manduca, A., Oliphant, T.E., Dresner, M.A., Mahowald, J.L., Kruse, S.A. et al.: Magnetic Resonance Elastography: Non-invasive mapping of Tissue Elasticity. Medical Image Analysis 5 (1997) 237-254

3. Knutsson, H., Westin, C.F., Granlund, G.: Local Multiscale Frequency and Bandwidth Estimation. In: Proceedings of IEEE Intl Conf on Image Processing (1994) 36-40

4. Oliphant, T.E., Manduca, A., Ehman, R.L., Greenleaf, J.F.: Complex-valued Stiffness Reconstruction for Magnetic Resonance Elastography. Magnetic resonance in Medicine 45 (2001) 299-310 